\title{
Cuticle and muscle variation underlying phenotypic plasticity in barnacle feeding leg and penis form
}

\author{
Christopher J. Neufeld ${ }^{1,2,3, a}$ and Cassidy Rankine ${ }^{1}$ \\ ${ }^{1}$ Department of Biological Sciences, University of Alberta, Edmonton, Alberta, T6G 2E9, Canada \\ ${ }^{2}$ Bamfield Marine Sciences Centre, Bamfield, British Columbia, V0R 1B0, Canada \\ ${ }^{3}$ Present address: Friday Harbor Laboratories, University of Washington, Friday Harbor, Washington, 98250, USA
}

\begin{abstract}
Many aspects of barnacle body form are known to be developmentally plastic. Perhaps the most striking examples of such plasticity occur in their feeding legs and unusually long penises, the sizes and shapes of which can change dramatically and adaptively with changes in conspecific density and local water flow conditions. However, whether variation in overall appendage form is mirrored by structural responses in cuticle and muscle is not known. In order to determine how structural variation underlies phenotypic plasticity in barnacle appendages, we examined barnacles occurring at low and high population densities from one wave-protected and one wave-exposed site. We used histological sectioning and fluorescence microscopy of feeding legs and penises to compare cuticle thickness, muscle thickness, and muscle organization, and artificial penis inflation to compare penis extensibility. We observed striking differences in cuticle thickness, muscle thickness, and muscle organization between sites that differed in water velocity, but we found no clear differences associated with variation in conspecific density. Penis extensibility also did not differ consistently between sites. These results are consistent with an adaptive explanation for much of the remarkable and complex variation in barnacle feeding leg and penis morphology among sites that differ in water velocity.
\end{abstract}

Additional key words: Balanus glandula, cirri, wave exposure

Rocky shores are among the most extreme and variable hydrodynamic environments on earth. On wave-exposed shores, the maximum velocity of breaking waves can exceed $25 \mathrm{~m} \mathrm{~s}^{-1}$, while in nearby protected bays, water velocity may be two orders of magnitude slower, never exceeding $10 \mathrm{~cm} \mathrm{~s}^{-1}$ (Denny 1988; Denny et al. 2003). Adding to this extreme spatial variability, water velocity at any one place can change dramatically as waves break and recede, and it may also vary significantly throughout the year due to the frequency and severity of storm events (Denny 1988). Surprisingly, despite this widespread variation in water velocity over space and time, a diverse assemblage of plants and animals thrives under these extreme conditions.

Intertidal barnacles are one particularly successful group that attains large population sizes on rocky shores worldwide. These small sessile crustaceans are filter feeders that use six pairs of modified

\footnotetext{
${ }^{\mathrm{a}}$ Author for correspondence.

E-mail: cneufeld@u.washington.edu
}

biramous thoracic legs, called cirri, to capture particles from the surrounding water. In most acorn barnacles, the last three pairs of cirri align to form a fan-like structure that is extended into the water column, while the first three pairs of cirri are used to filter smaller particles and to clean larger particles from the extended cirri (Anderson 1994). Barnacles extend these feeding legs by increasing hydrostatic pressure generated within a closed circulatory system, and retract them with a bundle of striated muscle fibers that runs from the base to the tip of each ramus (Anderson 1994). However, filter feeding poses a challenge to many intertidal barnacles, because they may face widely varying water velocities (Anderson 1994). On exposed shores, barnacle feeding legs must be able to withstand the force of breaking waves, while in quiet water, feeding legs must facilitate adequate water flow to capture enough food and facilitate gas exchange (Anderson 1994). Furthermore, feeding legs are not the only appendages that barnacles must extend into these extreme and variable flows. To reproduce, barnacles 
use impressively long (up to eight times their body length: Neufeld \& Palmer 2008) and dexterous penises to seek out and fertilize nearby mates (Klepal 1990). The penis is also extended through increased hydrostatic pressure, while its movements are controlled by striated longitudinal muscles that run the length of the penis along its outer wall (Klepal et al. 1972). Most barnacles are simultaneous hermaphrodites, so any neighbor may serve as a mate (Anderson 1994).

To cope with these challenges, many barnacles have evolved the capacity to change the sizes and shapes of their feeding legs and penises to suit water flow conditions (Arsenault et al. 2001; Marchinko 2003; Marchinko \& Palmer 2003; Hoch 2009). For example, in the Pacific acorn barnacle Balanus glandula DARWIN 1854, individuals transplanted to high flow grow feeding legs that are $\sim 50 \%$ shorter and $25 \%$ wider (Marchinko 2003) and penises that are $\sim 25 \%$ shorter and $50 \%$ wider (Neufeld \& Palmer 2008) for their length relative to individuals moved to calm water. In addition to these dramatic responses to changes in water velocity, barnacle feeding leg and penis form also vary with the density of nearby conspecifics. With few neighbors, barnacles have shorter feeding legs (Lopez et al. 2007; Neufeld 2011) and longer penises (Neufeld 2011). In this situation, shorter feeding legs may help barnacles cope with the reduced boundary layer, and longer penises may help them reach more distant neighbors. Further, members of at least one species respond to seasonal variation in wave force, growing shorter, stouter feeding legs, and wider penises during stormier winter months (C.J. Neufeld, unpubl. data).

Although appendage variation in barnacles has received growing attention in the last 10 years, all past studies of barnacle penis and leg form involve only measurements of overall form (of leg length and width, for example). Little is known about how individual components of these structures-e.g., cuticle and muscle - vary as overall form varies. Knowing whether changes in muscle and cuticle parallel functional changes in overall appendage form will help us understand the complexity and extent of form variation in acorn barnacle appendages, and may also shed light on trade-offs that could limit patterns of variation available to selection within and between environments.

In this study, we examined muscle and cuticle of barnacle feeding legs and penises because both are structural components that might affect a barnacle's ability to withstand high velocities on exposed shores. The total force generated by a muscle is proportional to its cross-sectional area (Vogel 2003). Therefore, we predicted that exposed-shore barnacles should have muscles with greater total cross-sectional area to control the feeding legs under the higher drag forces imposed by breaking waves. Further, striated muscle fibers are composed of repeating units known as sarcomeres, each made up of thick myosin and thin F-actin filaments that contract by sliding past each other (Huxley \& Hanson 1954; Huxley \& Niedergerke 1954). According to this sliding-filament model of muscle contraction, in the absence of other differences, longer resting sarcomeres should produce greater stress because the longer thick filaments they contain facilitate the formation of more actin-myosin cross-bridges acting in parallel. Conversely, muscles with shorter resting sarcomeres should produce greater maximum unloaded shortening velocity because more cross-bridges are acting in series. Indeed, resting sarcomere length scales isometrically with stress production across several animal groups, including crustaceans (Taylor 2000). In lobsters, longer sarcomeres $(>6 \mu \mathrm{m})$ are more often associated with fibers possessing higher endurance, while shorter sarcomeres $(<4 \mu \mathrm{m})$ are more often associated with fibers capable of greater shortening velocity (Jahromi \& Atwood 1971; Costello \& Govind 1983). Therefore, we made two contrasting predictions regarding sarcomere length in barnacle feeding leg muscles: (i) sarcomeres of exposed-shore barnacles may have longer sarcomeres to function better under the greater stresses experienced in high flow, and (ii) sarcomeres of exposed-shore barnacles may have shorter sarcomeres to allow feeding legs to retract more quickly when water velocities rise above the threshold for effective feeding (Miller 2007).

The cuticular exoskeleton is another important functional element of both feeding legs and penises. It not only provides structural support but also constrains internal fluid during hydrostatic extension of the penis and feeding legs. Therefore, we predicted that barnacles from an exposed shore should have thicker cuticle in both feeding legs and penises. Past measurements of penis form have been made on relaxed penises (Hoch 2008; Neufeld \& Palmer 2008; Hoch 2009; Neufeld 2011), and little is known about how much the penis can stretch (but see Neufeld \& Palmer 2008), or how the penis is able to function effectively when extended. Therefore, we also assessed whether patterns of variation observed in relaxed penises held true for extended penises. Finally, designing a structure that extends via hydrostatic pressure poses a significant material challenge. In a pressurized cylinder with a wall of 
uniform material properties, the circumferential stress is twice the longitudinal stress (Wainwright et al. 1982). In other words, any increase in pressure would result in a twofold increase in circumference for every unit increase in length. For a barnacle, the ability to counteract this circumferential expansion would increase penis extension at a given pressure and facilitate reaching more nearby mates. Therefore, we also studied how, and how well, barnacle penises are able to resist circumferential expansion during penis extension.

\section{Methods}

\section{Cuticle thickness and muscle cross-sectional area}

On 24 and 27 February 2009, individuals of Balanus glandula (mean soma mass $0.0321 \mathrm{~g}$, range 0.0152-0.068 g) were collected from two sites representing the extremes of a wave-exposure gradient in Barkley Sound, near Bamfield, British Columbia, Canada. The two sites were Bamfield Inlet (low velocity; $48.835382^{\circ},-125.136681^{\circ}$ ) and Seppings Island (high velocity; $48.841306^{\circ},-125.209525^{\circ}$ ); these sites are described by Neufeld \& Palmer (2008). The penis and $6^{\text {th }}$ left thoracic leg were removed at their bases from each individual and preserved in Bouin's solution for $24-28 \mathrm{~h}$ prior to being rinsed and moved to $70 \%$ ethanol before embedding. Samples were processed using a Leica Tissue Processor 1020 (Leica Microsystems Inc., Buffalo Grove, IL, USA) using a standard program for paraffin embedding. Briefly, samples were moved through a series of baths from $70 \%$ to $100 \%$ ethanol, then to toluene, and finally through to paraffin before being embedded in paraffin blocks for sectioning. Crosssections $5 \mu \mathrm{m}$ in thickness were taken at the base of the penis and feeding legs for muscle cross-section measurements. For the penis, the paraffin block was then reoriented and a longitudinal section was taken at the widest part of the penis, near its base. For the feeding legs, cross-sections were taken through the bases of both rami after they divided from the protopodite. All sections were mounted on glass slides using Mayer's fixative and stained with hematoxylin and eosin. Samples were mounted in DPX and photographed with a compound microscope at 100 $400 \times$ with a $6 \mathrm{MP}$ digital camera.

Structures were measured from photographs to the nearest $0.15 \mu \mathrm{m}$ using ImageJ (U.S. National Institutes of Health, Bethesda, MA, USA; http:// rsb.info.nih.gov/ij/). For penis cross-sectional muscle area, the area inside the centermost muscle bundles was subtracted from the area inside the outermost muscle bundles. Leg muscle cross-sectional area was calculated as the total muscle cross-sectional area (defined as the area between the outermost and innermost muscle bundles) of both rami divided by two. Leg cuticle thickness was measured at the outer (convex) margin of a single ramus, where the cuticle edges were typically the most well-defined. For penis cuticle measurements, data are means of three measurements taken at the convex outer margins of the three most intact and in-focus regions nearest the base of the penis. All photographs were measured without knowledge of collection location or conspecific density. To assess measurement error, we remeasured traits from photographs in ten randomly selected individuals. Repeatabilities (Whitlock \& Schluter 2009) varied from 0.93 to 0.99 depending on the trait. In other words, $93-99 \%$ of the variance in a trait was due to true differences, and only 1$7 \%$ was due to error in measuring photographs.

\section{Sarcomere length}

On 29 October 2009, individuals of B. glandula (mean soma mass $0.0191 \mathrm{~g}$, range $0.0021-0.0524 \mathrm{~g}$ ) were collected live with attached substrate from two additional sites in Barkley Sound. Wave-protected specimens were from Grappler Inlet $\left(48.832567^{\circ}\right.$, $-125.129796^{\circ}$ ) and wave-exposed individuals were from Wizard Island $\left(48.858144^{\circ},-125.160569^{\circ}\right)$. Barnacles were transported live to Edmonton, Alberta, and held in recirculating seawater aquaria at the University of Alberta. Mature individuals with aperture lengths between 3 and $5 \mathrm{~mm}$ were removed from their substrate and placed into $7 \%$ $\mathrm{MgCl}_{2}$ in seawater overnight (15-20 h) to relax muscles. Individuals were then removed from their shells and severed between the $3^{\text {rd }}$ and $4^{\text {th }}$ pairs of cirri with a scalpel under a dissecting microscope, being careful not to bend the cirral fan in order to prevent conformational changes to the sarcomeres. Tissues were then fixed in $5 \%$ paraformaldehyde overnight, then rinsed twice in $100 \mathrm{mmol} \mathrm{L}^{-1}$ phosphate buffered saline (PBS). Again using a scalpel and dissecting microscope, the $4^{\text {th }}$ and $6^{\text {th }}$ cirral pairs were severed below the protopodite, and the outer edge of the cuticle was split twice at the base, middle, and tip of each ramus to allow for actin label penetration. The cirri were then placed in $0.6 \mathrm{~mL}$ microcentrifuge tubes and permeabilized with $0.1 \%$ Triton X-100 (PBTX) for $10 \mathrm{~min}$ before adding $50 \mu \mathrm{L}$ of F-actin label (2 units Bodipy Fluorescein Phallacidin; $40 \mu \mathrm{L} 10 \%$ bovine serum albumin; $950 \mu \mathrm{L} \mathrm{PBTX).} \mathrm{Tissues} \mathrm{were} \mathrm{soaked} \mathrm{in} \mathrm{F-actin}$ label overnight, rinsed twice for $10 \mathrm{~min}$ in PBS, 
counterstained with $1 \mathrm{~mL}$ Hoechst 33342 (1 mmol $\mathrm{L}^{-1}$ final concentration) in PBTX for $1 \mathrm{~min}$, and then rinsed three more times in PBS. The cirri were then mounted on glass slides in Mowiol ${ }^{\circledR}$ mounting medium, with cover slips sealed with clear nail polish. Slides were stored in a refrigerator at $4^{\circ} \mathrm{C}$ and kept dark to prevent the fluorochromes from fading prior to viewing. Images were taken of the base, middle, and tip of each ramus using FITC and DAPI filters on a Zeiss Axioskop 2 Plus fluorescent microscope (Carl Zeiss Microscopy, LLC, Thornwood, NY, USA) with a QImaging QICAM CCD 10-bit digital camera (QImaging Inc., Surrey, BC, Canada) operated with Northern Eclipse software (Empix Imaging, Inc., Mississauga, ON, Canada).

Leg muscles remained attached to the cuticle during fixation and processing, and our method ensured that sarcomeres from all samples were in approximately the same resting state during imaging and measurement. Five sarcomeres were measured from each image using ImageJ, and the mean was used in all calculations. On each sarcomere, we measured thick filament (i.e., A-band) length as the distance between two successive I bands, a metric that should correlate with resting sarcomere length (Taylor 2000) and should be independent of slight conformational changes to the sarcomeres due to handling. Soma mass was back-calculated from measured leg length using ordinary least squares regressions in Marchinko \& Palmer (2003). Although this approach likely introduced some error, the tight correlation between body size and leg length at most sites suggests this approach is reasonable. Furthermore, the small effect of body size on sarcomere lengths $(3 \%$ in this study) suggests any errors in soma mass calculations should not appreciably influence results.

\section{Penis size and extensibility}

To investigate the overall form and material properties of barnacle penises, barnacles (mean soma mass $0.0398 \mathrm{~g}$, range $0.0106-0.0720 \mathrm{~g}$ ) were collected from the Bamfield Inlet and Seppings Island sites in areas of both low and high conspecific population densities. Approximately 15 barnacles were collected from each site and density combination in February 2009 and February 2010. Collected barnacles were immediately frozen for $>72 \mathrm{~h}$ before use. Prior to inflation, the soma was removed, and the penis was ablated, wet-mounted, and photographed using a 6-MP digital camera. The soma was then blotted dry and weighed to the nearest $0.01 \mathrm{mg}$.

For inflation, a thin capillary tube (PE 10) was inserted into the ablated end of the penis and care- fully glued in place with cyanoacrylate glue (Krazy Glue, Elmer's Products, Columbus, OH, USA) while keeping the penis tissue moist and ensuring that no air was allowed to enter into the system. The capillary tube was attached to a 30-gauge hypodermic needle connected to a $10-\mathrm{mL}$ serological pipette. The pipette was held using a micromanipulator to facilitate precise positioning of the setup during mounting and inflation. The other end of the pipette was attached to $10 \mathrm{~m}$ of flexible rubber tubing (4 $\mathrm{mm}$ internal diameter), which in turn was connected to a $100-\mathrm{mL}$ open-top bottle. The entire system was filled with seawater at ambient room temperature throughout the experiments. The bottle was capable of being raised to a height of $4.25 \mathrm{~m}$ above the tip of the needle using a pulley system, allowing pressure at the tip of the needle to be varied incrementally from ambient pressure $\leq 43 \mathrm{kPa}$ ( $\sim 6$ PSI). The penis was positioned in seawater on the stage of a dissecting microscope, and oriented parallel to the plane of view. As pressure in the system was increased at $\sim 2.5 \mathrm{kPa}$ intervals, the penis was photographed through the microscope using a 6-MP digital camera (after extension stabilized at each pressure interval) until maximum pressure was reached, or the penis ruptured. Penises were then measured to the nearest $10 \mu \mathrm{m}$ using ImageJ, as in Neufeld \& Palmer (2008).

The capacity to stretch was calculated as the change in dimension divided by the initial dimension and was computed for both longitudinal and circumferential changes during inflation. This is equivalent to calculating engineering strain in a homogenous material. However, we use the word "stretch" because the barnacle penis is bounded by cuticle containing many accordion-like folds (Fig. 1, inset) that unfold as the penis is pressurized. Therefore, "strain" seems misleading when including conformational changes of the cuticular folds themselves. However, we did estimate material stiffness of the penis cuticle. To do this, Young's modulus was calculated on stress-strain curves using pressures above $20 \mathrm{kPa}$ (data not shown), using the mean cuticle thickness for each exposure treatment (from Fig. 2A). This allowed us to estimate stiffness of the penis cuticle under biaxial strain at pressures where the cuticle was largely unfolded.

Inflation was attempted for all individuals from both years. However, due to the difficulty of working at this fine scale, penises were sometimes damaged during dissection and mounting. As a result, data from both years were combined in all analyses and sample sizes vary slightly. Furthermore, despite 

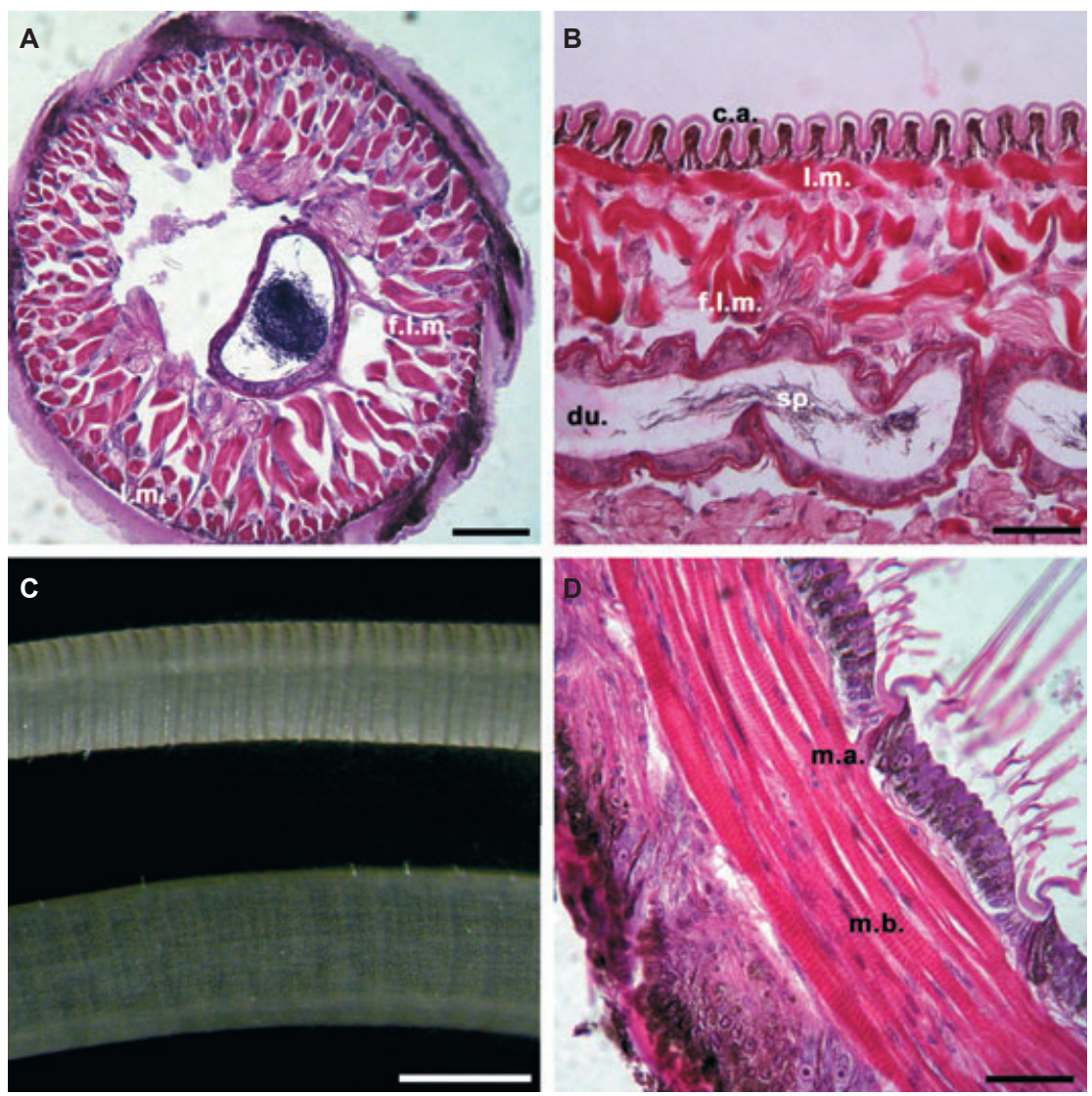

Fig. 1. Photomicrographs of the penis and feeding leg structure of the acorn barnacle Balanus glandula from histological sections and artificial penis inflation. A. Cross-section at the base of the penis of an exposed-shore barnacle (soma mass $=0.021 \mathrm{~g}$; opercular diameter $=5 \mathrm{~mm}$ ), showing longitudinal muscle bands $(1 \mathrm{~m}$.) in cross-section at outer edge, and in oblique section (f.l.m.) near the center. Scale bar $=0.05 \mathrm{~mm}$. B. Longitudinal section at the base of the penis of a protected-shore barnacle (soma mass $=0.022 \mathrm{~g}$; opercular diameter $=4.5 \mathrm{~mm}$ ), showing folded cuticular annulations (c.a.) at the outer edge, as well as taut longitudinal muscles (1.m.) near the edge, and loosely folded longitudinal muscle bands (f.l.m.) near the ductus (du.), which contains spermatozoa (sp.). Scale bar=0.05 mm. C. Base of a partially ( 100 Pa, top) and fully $(\sim 40 \mathrm{kPa}$, bottom) inflated penis of a protected-shore barnacle (soma mass $=0.023 \mathrm{~g}$; opercular diameter $=6 \mathrm{~mm}$ ), showing extension of annulations and increase in diameter with increased pressure. Scale bar $=0.5 \mathrm{~mm}$. $\mathbf{D}$. Longitudinal section near the middle of a feeding leg (single ramus) of an exposed-shore barnacle (soma mass=0.048 $\mathrm{g}$; opercular diameter $=6 \mathrm{~mm}$ ), showing clearly striated muscle bands (m.b.) of longitudinal retractor muscle, and muscle attachment (m.a.) at the upper of two visible articulations. Scale bar $=0.05 \mathrm{~mm}$.

being frozen for $>72 \mathrm{~h}$, some penises began moving autonomously during dissection. These samples were excluded from all analyses, except for data shown in Fig. 7. Remeasurement of ten randomly selected photographs revealed repeatabilities (Whitlock \& Schluter 2009) of 0.95 for penis length and 0.97 for penis basal diameter.

\section{Statistical analyses and calculations}

Analyses were conducted using R 2.10.1 (R Development Core Team 2010). To test the role of conspecific density and exposure on muscle crosssectional area and cuticle thickness of feeding legs and penises, and on relaxed and inflated penis form, we used ANCOVA on $\log _{10}$-transformed data using body size (soma mass) as a covariate. To test for differences in mean sarcomere length with exposure, feeding leg region, and leg number (i.e., leg 6 or leg 4), we used ANCOVA on $\log _{10}$-transformed data with body size (soma mass) as a covariate. Where appropriate, interaction terms were sequentially removed following Hendrix et al. (1982). Because the final model contained a significant leg number $\times$ body size interaction, terms involving leg number were also tested at one standard deviation above and below mean body size to determine if conclusions about sarcomere length between leg 4 and leg 
6 changed appreciably at different body sizes. Data conformed to assumptions of equal variance, and residuals were approximately normally distributed.

\section{Results}

\section{Structural organization}

In the penis, at least two distinct layers of longitudinal muscles were visible. The muscle bands that were closest to the epidermis were nearly circular in cross-section, and relatively straight in longitudinal section, while the inner muscle bands appeared more oval in penis cross-sections and appeared folded in the longitudinal sections of the relaxed penis (Fig. 1A,B). The annulations of the penis cuticle were clearly composed of accordion-like folds of relatively uniform thickness and were underlain by a layer of epidermal cells that also appeared folded in longitudinal sections through the relaxed penis (Fig. 1B). In the extended penis, annulations appeared completely unfolded (Fig. 1C). Longitudinal sections also revealed that the outer and folded inner longitudinal muscles of the penis were clearly attached to many cuticular annulations, though we could not determine from our sections whether muscles attached at every annulation. However, longitudinal sections through the feeding legs showed that the retractor muscle clearly attached to the cuticle at the base of each articulation (Fig. 1D). We also observed an obvious decrease in cuticle thickness moving from the external (nearest the anus) convex side to the inner (nearest the mouth) concave side of each ramus on all specimens (Fig. 2).

\section{Cuticle thickness and muscle cross-sectional area}

Barnacles from an exposed shore had cuticle that was $\sim 50 \%$ thicker in penises (Figs. 2, 3A), and $\sim 25 \%$ thicker in feeding legs (Fig. 3B), when compared with the cuticle of penises and feeding legs of similarly sized barnacles from a nearby protected harbor (Table 1). Mean penis and feeding leg muscle cross-sectional areas were $\sim 100 \%$ greater in barnacles from an exposed shore, compared with similarly sized barnacles from a nearby protected harbor (Fig. 3C,D; Table 1). However, cuticle thickness and muscle cross-sectional area in feeding legs and penises were not associated with variation in conspecific density (Table 1).

\section{Sarcomere length}

Sarcomere thick filaments (A-bands) at the bases of the feeding legs were more than twice as long as those at the tip (and were of intermediate length in
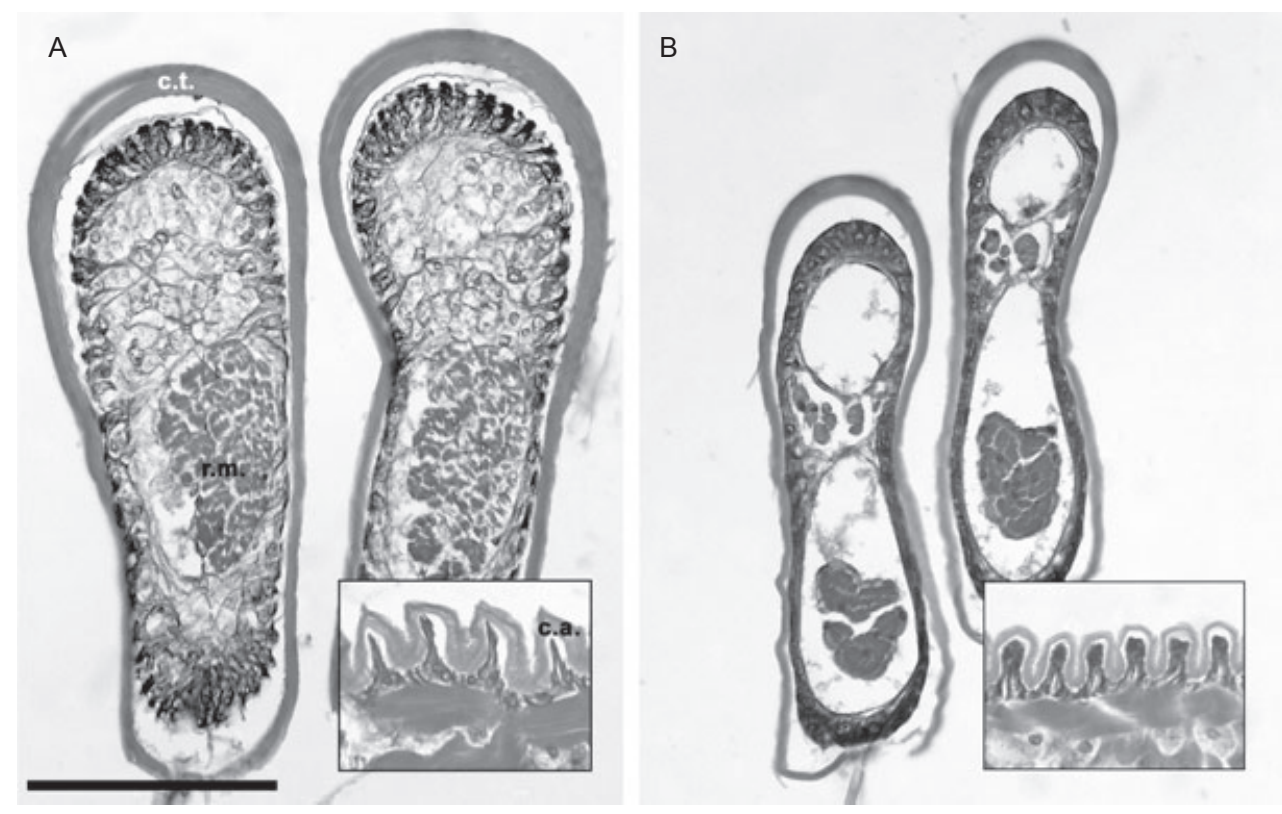

Fig. 2. Cross-sections of feeding legs (paired rami from leg six) and longitudinal sections of the penis near base (insets) from approximately equal-sized individuals of Balanus glandula (soma mass=0.024-0.026 g; opercular diameter $\sim 4.6 \mathrm{~mm}$ ) from Barkley Sound, British Columbia, Canada. A. Exposed-shore animal. B. Protected-shore animal. Scale bar $=0.1 \mathrm{~mm}$ for all images. Ca., cuticular annulations of penis; c.t., cuticle thickness at the outer convex (dorsal) surface of the feeding legs; r.m., retractor muscle. 

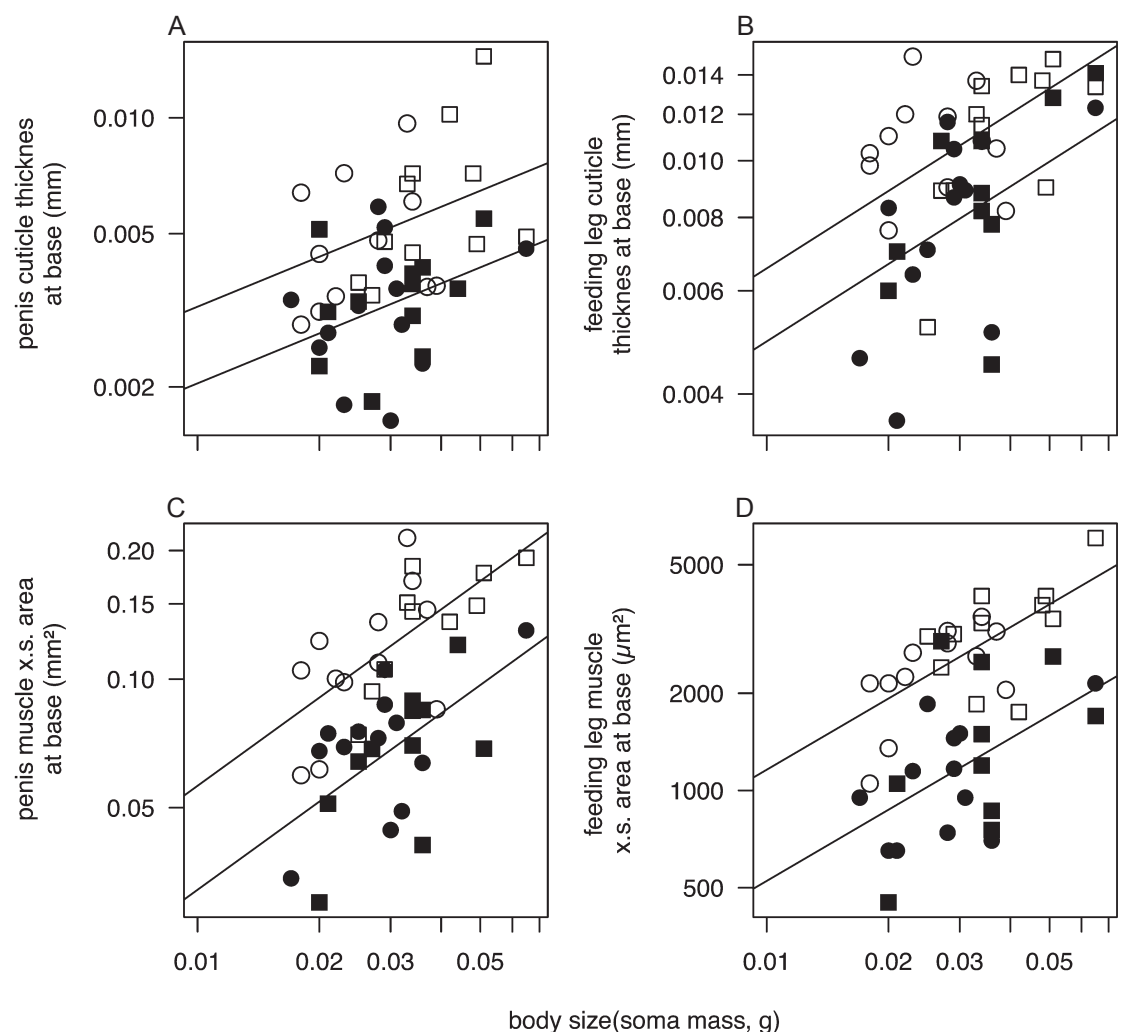

Fig. 3. Cuticle thickness $(\mathbf{A}, \mathbf{B})$ and total muscle cross-sectional area $(\mathbf{C}, \mathbf{D})$ of penises and feeding legs (mean for a single ramus of leg 6) of Balanus glandula as a function of body size (soma mass) from an exposed shore (open symbols) and a protected shore (closed symbols) in Barkley Sound, British Columbia, Canada. Squares represent barnacles with many neighbors; closed symbols represent barnacles with few neighbors. Lines represent best-fit regressions from final models (with few and many neighbors pooled at each site; Table 1).

the middle) for both feeding legs 4 and 6 , and leg region explained $>85 \%$ of the total variation in sarcomere length (Fig. 4; Table 2). Thick filament length was also associated with variation in wave exposure; barnacles from an exposed shore had $\sim 20 \%$ shorter thick filaments, compared with similarly sized barnacles from a nearby protected bay (Fig. 4A,B). This effect was consistent for both feeding legs 4 and 6 , although the magnitude of the effect differed slightly among leg regions (Table 2). Thick filament length also depended slightly on the two-way interaction between waveexposure and region, and the three-way interaction among leg number, region, and wave-exposure, though these effects were very small, explaining only $0.3 \%$ and $0.5 \%$ of total variation in sarcomere length, respectively. The final model (Table 2) also included a small but significant interaction between body size and leg number; subsequent analyses (Hendrix et al. 1982) show that the small significant effect of leg number is only present at smaller body sizes (Supporting Information, Fig. S1; Table S1).

\section{Penis size and extensibility}

Barnacles from a protected harbor had penises that were $\sim 25 \%$ longer than penises of barnacles from a nearby exposed shore; this pattern was present both before (Fig. 5A) and after (Fig. 5B) penis inflation. Barnacles with few reachable neighbors had $\sim 20 \%$ longer penises compared with barnacles with many reachable neighbors both before (Fig. 5A) and after (Fig. 5B) inflation, and this pattern was similar at both sites (Table 3). However, we observed no consistent differences in the capacity to stretch during inflation among penises of barnacles from sites with different water velocities or conspecific densities, despite more than threefold variation in the capacity for both longitudinal and circumferential stretch among individual barnacle penises (Fig. 6). Barnacle penises stretched approximately five times more in length than in circumference at maximum inflation (Figs. 6, 7). Pressure-stretch curves appear to decrease in slope at higher pressures (Fig. 6), though we observed no consistent differences in inflation curves among bar- 
Table 1. Results of final ANCOVAs on cuticle thickness and muscle cross-sectional area after all non-significant interactions were removed (results are illustrated in Figs. 2, 3). Significant effects are highlighted in bold.

\begin{tabular}{lccrr}
\hline \hline & $\mathrm{df}$ & $\mathrm{ms}$ & $\mathrm{F}$ & \multicolumn{1}{c}{$\mathrm{p}$} \\
\hline $\log _{10}$ (penis cuticle thickness at base) & & \\
$\log _{10}$ (body size) & 1 & 0.239 & 9.61 & $\mathbf{0 . 0 0 3 4}$ \\
Exposure & 1 & 0.459 & 18.42 & $<\mathbf{0 . 0 0 0 1}$ \\
Density & 1 & 0.004 & 0.17 & 0.6840 \\
Residuals & 43 & 0.025 & & \\
$\log _{10}$ (feeding leg cuticle thickness at base) & \\
$\log _{10}$ (body size) & 1 & 0.206 & 14.13 & $\mathbf{0 . 0 0 0 5}$ \\
Exposure & 1 & 0.174 & 11.98 & $\mathbf{0 . 0 0 1 3}$ \\
Density & 1 & 0.007 & 0.47 & 0.4983 \\
Residuals & 41 & 0.014 & & \\
$\log _{10}$ (penis muscle x.s. area at base) & & \\
$\log _{10}$ (body size) & 1 & 0.461 & 30.7 & $<\mathbf{0 . 0 0 0 1}$ \\
Exposure & 1 & 0.679 & 45.26 & $<\mathbf{0 . 0 0 0 1}$ \\
Density & 1 & 0.022 & 1.45 & 0.2355 \\
Residuals & 42 & 0.015 & & \\
$\log _{10}$ (feeding leg muscle x.s. area at base) & \\
$\log _{10}$ (body size) & 1 & 0.596 & 22.37 & $<\mathbf{0 . 0 0 0 1}$ \\
Exposure & 1 & 1.332 & 50.79 & $<\mathbf{0 . 0 0 0 1}$ \\
Density & 1 & 0.007 & 0.27 & 0.6061 \\
Residuals & 41 & 0.026 & & \\
\hline
\end{tabular}

nacles from different water velocities and conspecific densities (Fig. 7). Under biaxial strain, the Young's modulus above $20 \mathrm{kPa}$ (mean \pm standard error) for circumferential stretch was nearly tenfold higher $(40.57 \pm 3.0 \mathrm{MPa})$ than for longitudinal stretch $(4.36 \pm 0.43 \mathrm{MPa})$. Body size was not related to maximum longitudinal or circumferential stretch $(\mathrm{p}>0.61)$. Also, penises of exposed-shore barnacles showed no clear evidence of being able to sustain higher pressure before rupturing, despite having 50\% thicker cuticle (Fig. 2A) relative to similarly sized protected-shore individuals. Of the 14 protected-shore penises and ten exposedshore penises that ruptured before maximum experimental pressure was reached, only five penises each of exposed-shore and protected-shore barnacles ruptured beyond the base, and thus can be attributed to a natural failing of the cuticle rather than to artefacts of the attachment procedure. Of these "natural" failure events, there was no significant difference in pressure to rupture between exposed-shore (mean $=37 \mathrm{kPa}$ ) and protectedshore $($ mean $=32 \mathrm{kPa})$ barnacle penises $(t=1.3804$, $\mathrm{p}=0.222$ ). Penises with living muscle tissue omitted from earlier analyses showed less longitudinal extension at low pressures $(\sim 0-20 \mathrm{kPa})$, compared with penises of barnacles with inactive muscle tissue, but converged at approximately equal extension at higher pressures ( $\sim 20-25 \mathrm{kPa}$; Fig. 8A). We did not observe any obvi-
Table 2. Results of final ANCOVA on thick filament length after all non-significant higher-order interactions were removed (results are illustrated in Fig. 4). Significant effects are highlighted in bold. In this analysis, "leg" refers to whether samples were taken from the fourth or sixth feeding leg pair; "region" refers to where on the feeding leg measurements were made (base, middle, or tip).

\begin{tabular}{llcccc}
\hline \hline & \multicolumn{5}{c}{$\log _{10}$ (thick filament length) } \\
\cline { 2 - 6 } & df & $\mathrm{ms}$ & \multicolumn{1}{c}{$\mathrm{F}$} & $\mathrm{p}$ & $R^{2}$ \\
\hline $\log _{10}$ (body size) & 1 & 0.12 & 80.52 & $\mathbf{< \mathbf { 0 . 0 0 1 }}$ & 0.031 \\
(S) & & & & & \\
Exposure (E) & 1 & 0.12 & 80.71 & $<\mathbf{0 . 0 0 1}$ & 0.031 \\
Leg (L) & 1 & 0.03 & 19.41 & $<\mathbf{0 . 0 0 1}$ & 0.008 \\
Region (R) & 2 & 1.68 & 1139.18 & $<\mathbf{0 . 0 0 1}$ & 0.862 \\
E $\times$ L & 1 & 0 & 0.43 & 0.514 & 0.000 \\
E $\times$ R & 2 & 0.01 & 5.52 & $\mathbf{0 . 0 0 5}$ & 0.005 \\
L $\times$ R & 2 & 0.02 & 13.7 & $<\mathbf{0 . 0 0 1}$ & 0.010 \\
S $\times$ L & 1 & 0.01 & 7.11 & $\mathbf{0 . 0 0 8}$ & 0.003 \\
E $\times$ L $\times$ R & 2 & 0.01 & 3.7 & $\mathbf{0 . 0 2 8}$ & 0.003 \\
Residuals & 130 & 0.001 & & & \\
\hline
\end{tabular}

ous differences in circumferential inflation curves between living and dead tissue (Fig. 8B).

\section{Discussion}

We identified a number of traits in barnacle feeding legs and penises that vary as expected from predictions from engineering theory, and that are consistent with adaptation to the increased hydrodynamic stress encountered on wave-exposed shores. Specifically, to cope with the extreme forces imposed by breaking waves on exposed shores (Denny 2006), barnacle feeding legs and penises must meet two important criteria: (i) feeding legs and penises must resist deformation and structural failure under applied loads, and (ii) they must possess muscles strong enough to control these structures in the extreme and variable flows they routinely experience. Below we address each of these in turn.

\section{Thicker cuticle should help resist deformation}

In feeding legs, the $25 \%$ thicker cuticle of waveexposed individuals should significantly increase resistance to compression and to local buckling under load. When the feeding fan is oriented into flow, drag exerted on the feeding legs puts the inside surface in tension, while the outer surface is put in compression. Therefore, the thicker cuticle on the outer surface of the feeding legs should distribute compression forces over a greater cross-sectional 

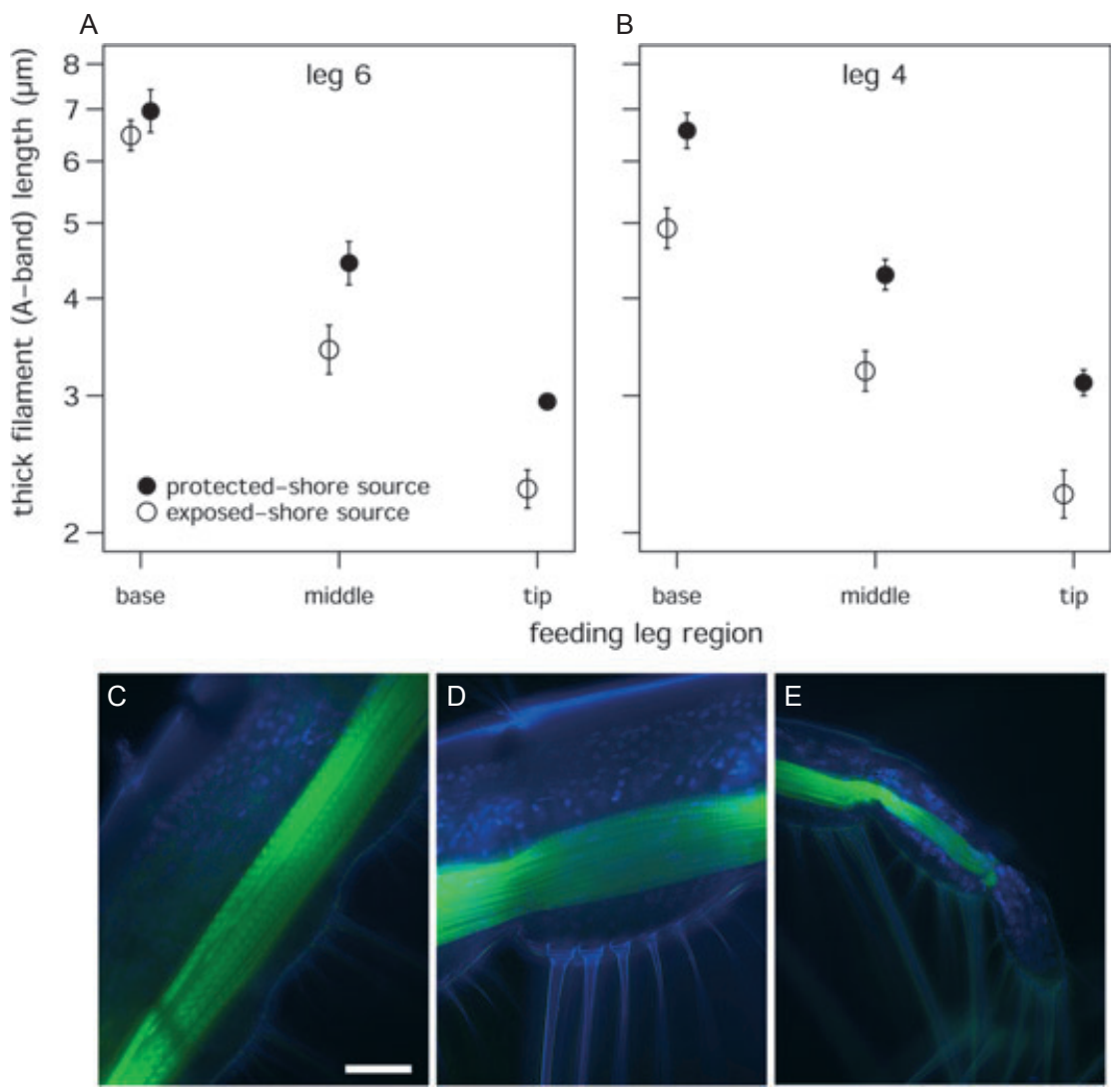

Fig. 4. Thick filament length (A-band length) of feeding-leg retractor muscles of the acorn barnacle Balanus glandula. A, B. Mean body-size-adjusted thick filament length from leg 6 (A) and leg 4 (B) (representing the medial- and lateralmost feeding legs of the cirral fan, respectively) compared across leg regions from an exposed-shore (open symbols) and a protected shore (closed symbols). All measurements were standardized to an average-sized barnacle (soma wet mass $=0.0125 \mathrm{~g}, \sim 4 \mathrm{~mm}$ opercular diameter) using ANCOVA (Table 2). C-E. Representative fluorescence microscopy images taken at the base (C), middle (D), and tip (E) of a barnacle feeding leg (leg 6). The labelled F-actin of the retractor muscle is shown in green. Scale bar $=50 \mu \mathrm{m}$ for $\mathrm{C}-\mathrm{E}$.

Table 3. Results of final ANCOVAs on relaxed and inflated length after all non-significant interactions were removed (results are illustrated in Fig. 5). Significant effects are highlighted in bold.

\begin{tabular}{lccrr}
\hline \hline & $\mathrm{df}$ & $\mathrm{ms}$ & $\mathrm{F}$ & \multicolumn{1}{c}{$\mathrm{p}$} \\
\hline $\log _{10}$ (relaxed penis length) & & & \\
$\log _{10}$ (body size) & 1 & 0.004 & 1.72 & $\mathbf{0 . 0 1 9 6}$ \\
Exposure & 1 & 0.034 & 14.20 & $\mathbf{0 . 0 0 0 5}$ \\
Density & 1 & 0.031 & 13.16 & $\mathbf{0 . 0 0 0 7}$ \\
Residuals & 47 & 0.111 & & \\
$\log _{10}$ (extended penis length) & & & \\
$\log _{10}$ (body size) & 1 & 0.011 & 3.93 & 0.0534 \\
Exposure & 1 & 0.082 & 30.11 & $<\mathbf{0 . 0 0 0 1}$ \\
Density & 1 & 0.045 & 16.49 & $\mathbf{0 . 0 0 0 2}$ \\
Residuals & 47 & 0.128 & & \\
\hline
\end{tabular}

area and increase the second moment of area, thereby allowing the feeding legs to withstand higher drag without buckling. More generally, the allocation of thicker cuticle on the posterior side of the feeding legs in both wave-exposed and wave-protected individuals (Fig. 2) should increase strength while economizing on material on the inside surface of the feeding legs, where the cuticle should be under tension when the feeding fan is extended in flow.

In the penis, the thicker cuticle of wave-exposed individuals should also reduce the risk of structural failure, but for a slightly different reason. Unlike the feeding legs, which must resist deformation largely in one direction, the extended penis must cope with forces in all directions. Thicker cuticle should help barnacle penises resist the larger forces on wave-exposed shores for two reasons, both related to local buckling. When a bending force is exerted on a cylinder, the material on one side is put in tension, while the other side is put in compression. This has two important implications for barnacle penises. First, at any given pressure, thicker cuticle should increase the force required to initiate buckling under 

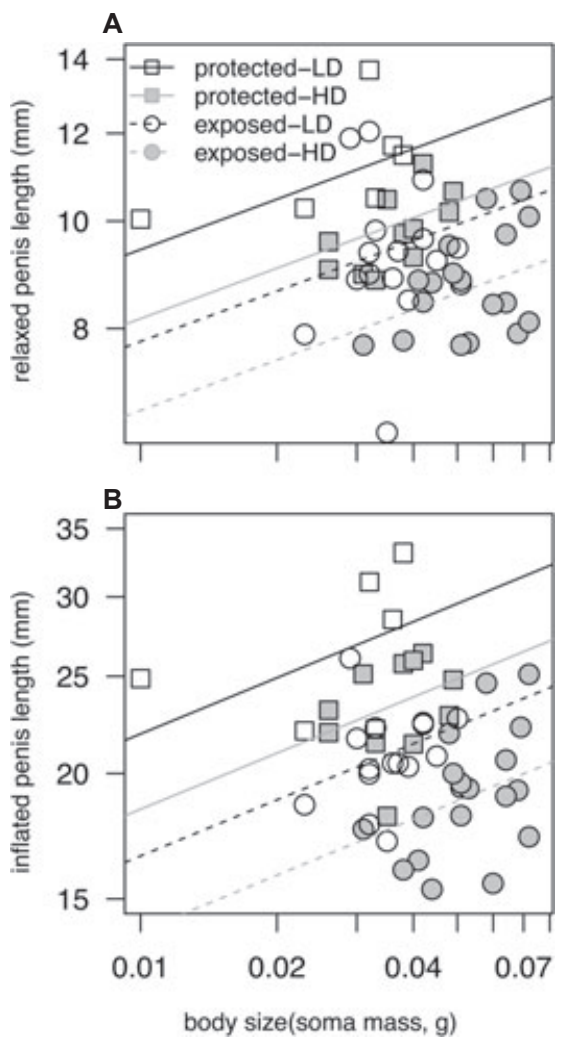

Fig. 5. Relaxed (A) and inflated (B) penis length before and after artificial inflation for Balanus glandula from low and high conspecific density at a wave-exposed shore and a protected shore in Barkley Sound, British Columbia, Canada. Lines represent best-fit regressions from final models after removing all non-significant interactions (Table 3). Squares and solid lines are protected-shore barnacles, circles and dotted lines are exposed-shore barnacles; open symbols and black lines are barnacles with few neighbors, grey symbols and lines are barnacles with many neighbors.

compression that will ultimately result in a sharp bending and lack of proper penis function (Kelly 2007), as sometimes seen in copulating dogs (Grandage 1972). Second, and perhaps more importantly, because wall stress is inversely related to wall thickness (Wainwright et al. 1982), thicker cuticle should be able to withstand greater internal pressures before breaking. Although we found only a small and non-significant difference in mean pressure-torupture between exposed-shore and protected-shore barnacle penises, this may be an artefact of the experimental procedure. Specifically, we excluded all rupture events where cuticle failed at the base, because we could not separate failures due to damage during mounting from true failure of the cuticle. In other words, the sample we used was biased to include only barnacles whose penises ruptured near the tip.
However, the idea that the penises of barnacles from exposed shores can and do operate at higher internal pressures than those of barnacles from protected shores is supported by one additional observation. When we consider all failure events (including those where the penis ruptured at the point of attachment), protected-shore barnacle penises generally failed at lower pressures (Fig. 6). If exposed-shore barnacle penises are indeed able to withstand and function at higher internal pressures, this increased pressure should act to resist the inward force at a local bend and thereby further decrease the risk of local buckling and failure. The thicker cuticle of exposed-shore barnacle penises may have one additional benefit as well. The edges of the shells of barnacles are quite sharp. Therefore, the thicker cuticle may reduce the risk of damage to penises during mating in the higher flow experienced on wave-exposed shores.

Collectively, when the results reported here are considered with previous work documenting an $\sim 20 \%$ increase in basal diameter of feeding legs and penises on exposed shores (Marchinko \& Palmer 2003; Neufeld \& Palmer 2008), the likely increase in bending stiffness of feeding legs and penises from exposed shores becomes even more significant. Specifically, the second moment of area (which is proportional to bending stiffness) of a cylinder scales with the radius to the fourth power, and also increases as new material is added at greater distances from the neutral axis (Vogel 2003). For example, for a cylinder, a $20 \%$ increase in diameter would result in a $31 \%$ increase in flexural stiffness, while a $25 \%$ increase in wall thickness (assuming new material is added on the outside) would increase flexural stiffness by $30 \%$. However, taken together, a $25 \%$ thicker wall and a $20 \%$ greater diameter would increase flexural stiffness by $70 \%$. Although feeding legs and penises are clearly structurally more complex than simple cylinders, this simplistic example nonetheless suggests that changes in structure of feeding legs and penises seen on exposed shores should significantly increase flexural stiffness. This is seen in the ability of exposed-shore barnacles to extend their feeding legs in higher flows in the laboratory (Marchinko 2007), and in the increased fertilization success on exposed shores of barnacles with wider penises (Hoch 2009). When combined with the predicted decrease in drag of shorter feeding legs (Marchinko \& Palmer 2003) and shorter penises (Neufeld \& Palmer 2008), barnacles on exposed shores appear remarkably well adapted for life in these conditions. 

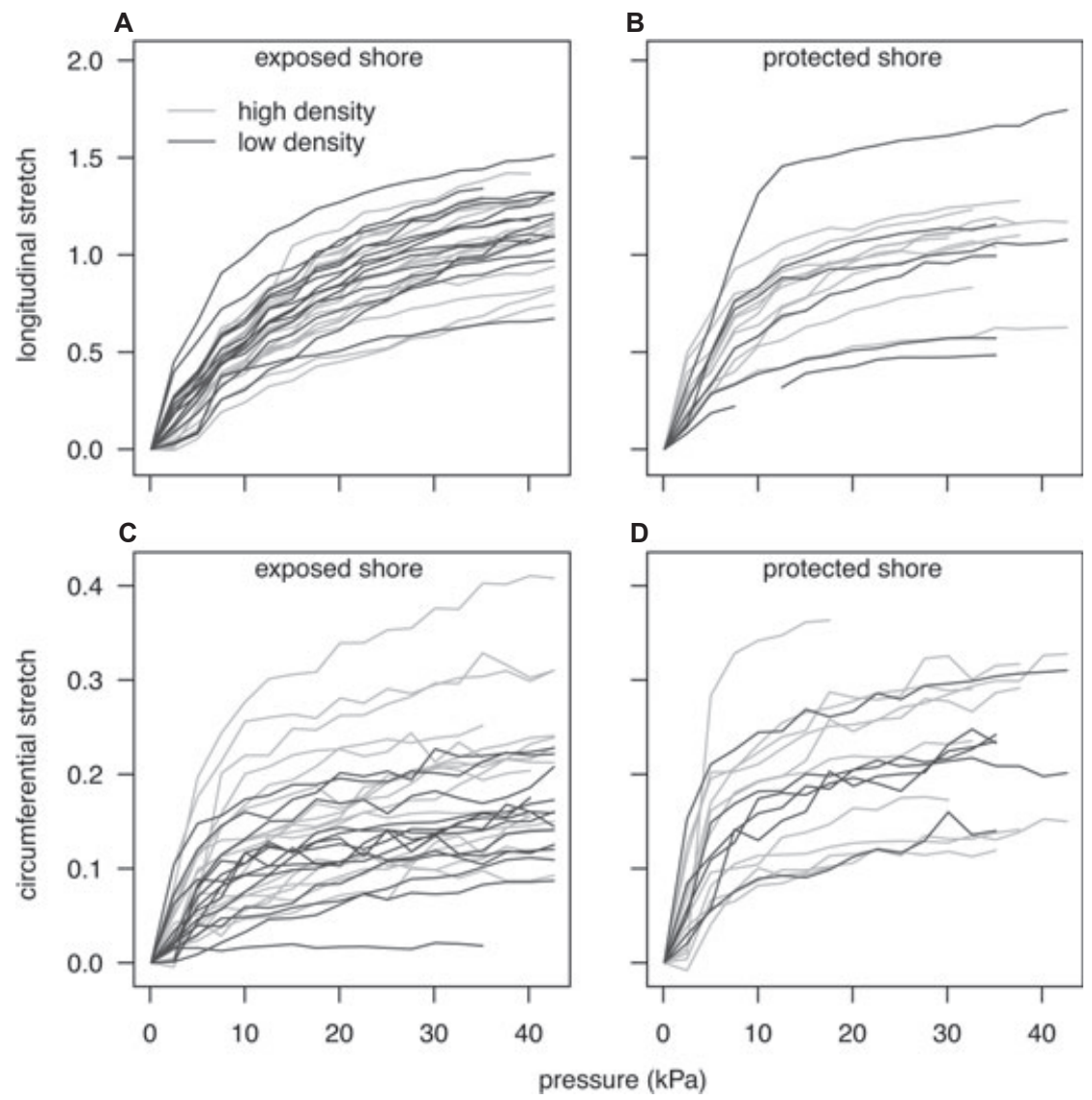

Fig. 6. Standardized barnacle penis form during artificial inflation. A, B. Longitudinal stretch as a function of internal pressure. C, D. Circumferential stretch as a function of internal pressure. Acorn barnacles (Balanus glandula) from both high and low conspecific densities were collected from an exposed shore $(\mathrm{A}, \mathrm{C})$ and a protected shore (B, D) in Barkley Sound, British Columbia, Canada. Lines represent curves for individual barnacles.

\section{Significance of variation in muscle form}

In addition to resisting deformation, to feed and mate on wave-exposed shores, barnacle feeding legs and penises must have muscles strong enough to effectively control these appendages in the face of the higher forces imposed on them in these highenergy environments. The increases we observed in cross-sectional area of muscles in the feeding legs and penises of barnacles from the wave-exposed site should allow them to do just this. Specifically, because the force generated by a muscle is proportional to its cross-sectional area, a doubling of cross-sectional area should equate to a twofold increase in total force production.

We also tested the prediction that resting sarcomeres (and corresponding thick filaments) should be longer on exposed shores to control the feeding legs under increased drag (recall that longer resting sarcomeres are associated with greater force production in crustaceans; Costello \& Govind 1983;
Taylor 2000). Contrary to our predictions, however, we observed $20 \%$ shorter thick filaments in sarcomeres of wave-exposed individuals, relative to equal-sized wave-protected counterparts. One possible explanation for this unexpected result involves the interaction between feeding behavior and another trait often correlated with resting sarcomere length, muscle endurance. In crustaceans, muscles with longer sarcomeres are generally composed of fibers with high endurance, while muscles with shorter sarcomeres often contain more aerobic muscle tissue better for short bursts of activity (Jahromi \& Atwood 1971; Costello \& Govind 1983; Vogel 2003). Because wave-protected individuals feed with a fast and relatively constant cirral beat of $\sim 20-40$ beats per minute, while wave-exposed forms typically feed by holding the cirral net steady and taking advantage of passive flow generated by breaking waves (Anderson 1981; Marchinko 2007), wave-protected individuals should benefit from a greater concentration of high-endurance muscle 


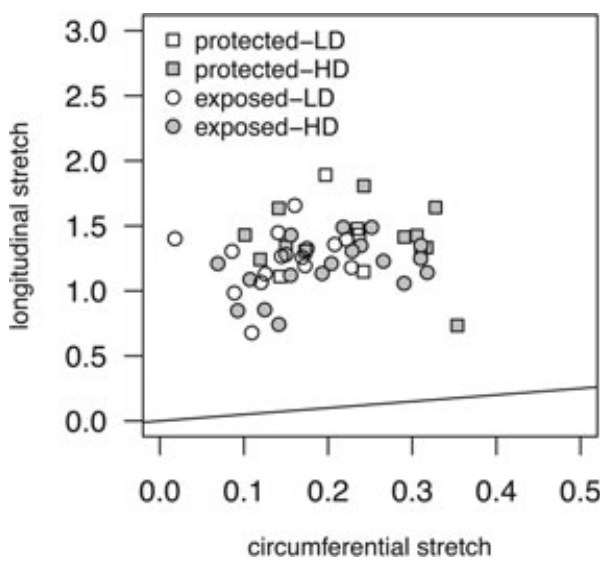

Fig. 7. Standardized longitudinal extension (stretch) as a function of circumferential stretch during artificial penis inflation at maximum pressure $(43 \mathrm{kPa})$ for Balanus glandula. Symbols represent penises of barnacles from both high (squares) and low (circles) conspecific densities from an exposed shore (open) and a protected shore (grey) in Barkley Sound, British Columbia, Canada. Each point represents an individual barnacle. The line represents the prediction for a pressurized cylinder whose walls are composed of an isotropic material.

fibers to facilitate the higher sustained rate of cirral beating.

Furthermore, when the variation in sarcomere length is considered with the parallel increase in muscle cross-sectional area, the combination of more muscle with shorter resting sarcomeres on exposed shores may in fact be an elegant solution to the need for both quick and strong muscles in these habitats. Specifically, the increased speed from shorter sarcomeres may allow for the quick and carefully timed movement of feeding legs required to dodge the largest waves on exposed shores (Miller 2007), while the increase in muscle cross-sectional area should maximize total force production to counteract the greater forces experienced by the feeding fan in breaking waves.

We also found $\sim 50 \%$ shorter thick filaments at the tips of the feeding legs compared with the bases. Although this variation was unexpected, two plausible adaptive explanations, acting either alone or in concert, could account for this striking pattern. If the shorter thick filaments result in a greater maximum unloaded shortening velocity for the muscle (Jahromi \& Atwood 1971; Costello \& Govind 1983), increased retraction speed at the tip of the cirri could prevent captured food from flowing out past the top of the fan before transfer to the maxillipeds on the forward sweep of the cirral beat. This increased speed could also be adaptive against predators. Barnacle cirri are frequently grazed upon by
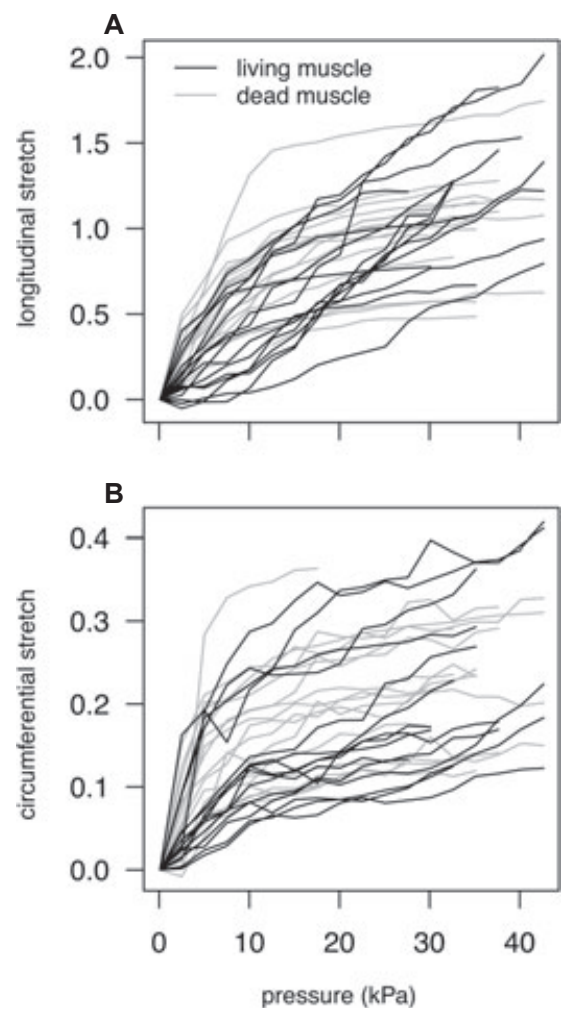

Fig. 8. Standardized penis wall extension (stretch) during artificial inflation of penises of Balanus glandula. A. Longitudinal stretch as a function of internal pressure. B. Circumferential stretch as a function of internal pressure. Black lines represent additional barnacles excluded from earlier analyses because penis tissue was still living during inflations (see Methods).

fish and other predators (Harvey et al. 2003), so faster retraction speeds at the tip of the feeding legs could help avoid grazing by fish. Further, presuming muscles with longer thick filaments are stronger (Costello \& Govind 1983; Taylor 2000), greater force generation at the base of the feeding legs may help to counteract the greater cumulative drag force experienced in this leg region (the drag force exerted at the base of the feeding legs should be greatest because the entire leg is exerting force at the base, while the tip experiences drag only from the distal region). Given these clear functional predictions of variation in maximum unloaded shortening velocity, one promising avenue for future research would be to use high-speed video to quantify leg retraction speeds along the length of the feeding legs from exposed-shore and protected-shore barnacles.

\section{Mechanics of penis inflation}

Changes in length and basal diameter of relaxed and extended penises were consistent with each 
other and with results of past studies. This confirms that conclusions from two previous studies of the form of relaxed penises of Balanus glandula (Neufeld \& Palmer 2008; Neufeld 2011) should apply to extended penises as well. However, despite documenting for the first time clear variation in extended penis form among sites and densities in $B$. glandula, we did not see any difference in the ability to stretch of penises from different wave-exposure environments or densities. This suggests that variation in structure rather than variation in material properties (of the cuticle, for example) accounts for variation in performance among wave exposures and population densities.

Barnacle penises stretched a remarkable five times more in length than in circumference, likely a result of the well-defined cuticular annulations that run the length of the penis (Fig. 1B). Much like the folds of an accordion, these annulations should allow the penis to stretch along its length while resisting circumferential expansion. The observed increase in stiffness at higher pressures (exemplified by a decrease in extension for a given increase in pressure; Fig. 8) is consistent with a dominant role of cuticular folds in facilitating longitudinal extension. This is also supported by the nearly tenfold higher circumferential stiffness relative to longitudinal stiffness of the penis during inflation. Penises of other animals have traits that appear to serve a similar function. For example, the penises of armadillos (Kelly 1999a) and turtles (Kelly 1999b, 2004) are inflatable structures surrounded by an axial-orthogonal array of collagen fibers. When the penis is relaxed, the fibers are folded, and when the penis extends, these fibers straighten out (Kelly 1997, 2004), resulting in a sharp increase in stiffness at maximum strain (Kelly 1999a). In barnacles, the folded cuticle likely serves a similar function (Fig. 1).

We also identified an unusual organization of muscle bands that should allow the penis to remain moveable and flexible at different extensions. Our histological sections clearly showed multiple muscle bands attached to the cuticle, and some muscle bands were more folded than others in the relaxed penis. Such an array of muscle bands of different lengths would allow different muscle bands to be used for penis control as the penis stretches, without individual muscle bands needing to operate across a wide range of extensions. This organization of muscles was not noted in a previous study of penis histology in another barnacle species (Semibalanus balanoides (LinNaEus 1758): Klepal et al. 1972), so it is unclear how many barnacles possess such a muscle organization.

The barnacle penis clearly faces an unusual set of functional requirements. Not only must it be retract- able to be stored safely and compactly when not in use, it also must remain strong, flexible, and moveable while extending across a wide range of distances from the animal. Above, we have identified numerous features that may help barnacles meet these unusual requirements. However, a number of future studies would be fruitful. For example, although differences in penis inflation curves between living and dead muscle offer some clue about the range of pressures at which the penis functions, comparison of inflation curves of penises with chemically relaxed and fully contracted muscle using the procedure outlined in Hoyle \& Smyth (1963) could allow estimates of maximum penis muscle strength from barnacles living in different population densities and habitats, and would also confirm the range of pressures under which barnacle penises are capable of functioning. Further, to complement laboratory studies, direct measurement of in vivo pressures of barnacles from exposed and protected shores could directly document the pressures required to extend penises in the field.

Acknowledgments. We thank S. Leys for feedback and assistance with the fluorescent labelling, staining, and histological sectioning; A.R. Palmer for help with collections, sarcomere measurements, and data analysis; J. Gosline, M. Denny, and E. Carrington for comments on penis inflation biomechanics; C. Gruman for help in the field; and A. Oatway and the University of Alberta's Advanced Microscopy Facility for help with histological work and fluorescence microscopy. This work was funded by a NSERC PGS-D Scholarship and an Alberta Ingenuity Fund Award to C.J.N., a NSERC Discovery Grant to S. Leys, and a NSERC Discovery grant (A7245) to A.R. Palmer. Comments from A.R. Palmer, H. Proctor, B. Pernet, and two anonymous reviewers greatly improved this manuscript.

\section{References}

Anderson DT 1981. Cirral activity and feeding in the barnacle Balanus perforatus Bruguiere (Balanidae), with comments on the evolution of feeding mechanisms in thoracican Cirripedes. Philos. Trans. R. Soc. Lond. B Biol. Sci. 291: 411-449.

1994. Barnacles: Structure, Function, Development and Evolution. First edition. Chapman \& Hall, London.

Arsenault DJ, Marchinko KB \& Palmer AR 2001. Precise tuning of barnacle leg length to coastal wave action. Proc. R. Soc. Lond. B Biol. Sci. 268: 2149-2154.

Costello WJ \& Govind CK 1983. Contractile responses of single fibers in lobster claw closer muscles - correlation with structure, histochemistry, and innervation. J. Exp. Zool. 227: 381-393. 
Denny MW 1988. Biology and the Mechanics of the Wave-Swept Environment. Princeton University Press, Princeton, NJ.

2006. Ocean waves, nearshore ecology, and natural selection. Aquat. Ecol. 40: 439-461.

Denny MW, Miller LP, Stokes MD, Hunt LJH \& Helmuth BST 2003. Extreme water velocities: topographical amplification of wave-induced flow in the surf zone of rocky shores. Limnol. Oceanogr. 48: 1-8.

Grandage J 1972. Erect dog penis - paradox of flexible rigidity. Vet. Rec. 91: 141-147.

Harvey R, Burrows MT \& Speirs R 2003. Cirral regeneration following non-lethal predation in two intertidal barnacle species. J. Mar. Biol. Assoc. UK 83: 1229-1231.

Hendrix LJ, Carter MW \& Scott DT 1982. Covariation analyses with heterogeneity of slopes in fixed models. Biometrics 38: 641-650.

Hoch JM 2008. Variation in penis morphology and mating ability in the acorn barnacle, Semibalanus balanoides. J. Exp. Mar. Biol. Ecol. 359: 126-130.

2009. Adaptive plasticity of the penis in a simultaneous hermaphrodite. Evolution 63: 1946-1953.

Hoyle G \& Smyth T 1963. Giant muscle fibers in a barnacle, Balanus nubilus Darwin. Science 139: 49-50.

Huxley H \& Hanson J 1954. Changes in the cross-striations of muscle during contraction and stretch and their structural interpretation. Nature 173: 973-976.

Huxley AF \& Niedergerke R 1954. Structural changes in muscle during contraction: interference microscopy of living muscle fibres. Nature 173: 971-973.

Jahromi SS \& Atwood HL 1971. Structural and contractile properties of lobster leg muscle fibers. J. Exp. Zool. 176: 475-486.

Kelly DA 1997. Axial orthogonal fiber reinforcement in the penis of the nine-banded armadillo (Dasypus novemcinctus). J. Morphol. 233: 249-255.

- 1999a. Expansion of the tunica albuginea during penile inflation in the nine-banded armadillo (Dasypus novemcinctus). J. Exp. Biol. 202: 253-265.

1999b. Axial orthogonal fiber arrays in the penis of the loggerhead turtle (Caretta caretta). Am. Zool. 39: 569 .

2004. Turtle and mammal penis designs are anatomically convergent. Proc. R. Soc. Lond. B Biol. Sci. 271: S293-S295.

2007. Penises as variable-volume hydrostatic skeletons. In: Reproductive Biomechanics. Elad D \& Young RC, eds., pp. 453-463. Wiley-Blackwell, New York.

Klepal W 1990. The fundamentals of insemination in cirripedes. Oceanogr. Mar. Biol. 28: 353-379.

Klepal W, Barnes H \& Munn EA 1972. The morphology and histology of the cirripede penis. J. Exp. Mar. Biol. Ecol. 10: 243-265.

Lopez BA, Guaitro SY \& Lopez DA 2007. Densitydependent effects on the cirral structure of the barnacle, Jehlius cirratus (Darwin, 1854) (Cirripedia). Crustaceana 80: 793-801.
Marchinko KB 2003. Dramatic phenotypic plasticity in barnacle feeding legs (Balanus glandula Darwin): magnitude, age dependence, and speed of response. Evolution 57: 1281-1290.

2007. Feeding behavior reveals the adaptive nature of plasticity in barnacle feeding limbs. Biol. Bull. 213: $12-15$.

Marchinko KB \& Palmer AR 2003. Feeding in flow extremes: dependence of cirrus form on wave-exposure in four barnacle species. Zoology 106: 127-141.

Miller LP 2007. Feeding in extreme flows: behavior compensates for mechanical constraints in barnacle cirri. Mar. Ecol. Prog. Ser. 349: 227-234.

Neufeld CJ 2011. Modular phenotypic plasticity: divergent responses of barnacle penis and feeding leg form to variation in density and wave exposure. J. Exp. Zool B Mol. Dev. Evol. 316B: 254-262 doi: 10.1002/jez. b. 21395

Neufeld CJ \& Palmer AR 2008. Precisely proportioned: intertidal barnacles alter penis form to suit coastal wave action. Proc. R. Soc. Lond. B Biol. Sci. 275: 1081 $-1087$.

R Development Core Team 2010. R: A Language and Environment for Statistical Computing. R Foundation for Statistical Computing, Vienna, Austria.

Taylor GM 2000. Maximum force production: why are crabs so strong? Proc. R. Soc. Lond. B Biol. Sci. 267: 1475-1480.

Vogel S 2003. Comparative Biomechanics: Life's Physical World. Princeton University Press, Princeton, NJ.

Wainwright SA, Biggs WD, Currey JD \& Gosline JM 1982. Design Mechanical in Organisms. Press Princeton University, Princeton, NJ.

Whitlock MC \& Schluter D 2009. Analysis the of Data Biological. Roberts and Company, Village Greenwood, CO.

\section{Supporting information}

Additional Supporting information may be found in the online version of this article:

Figure S1. Feeding-leg thick filament (A-band) length of Balanus glandula adjusted to (A, B) one standard deviation below the mean body size (soma mass), (C, D) mean body size, and (E, F) one standard deviation above the mean body size.

Table S1. Adjusted ANCOVA on thick filament (A-band) length, at one standard deviation below the mean body size (soma mass), at the mean, and at one standard deviation above the mean (results are illustrated in Fig. S1).

Please note: Wiley-Blackwell is not responsible for the content or functionality of any supporting materials supplied by the authors. Any queries (other than missing material) should be directed to the corresponding author for the article. 